\title{
Method of Power Generation after Hydrogen is Produced with Piggery Wastewater
}

\author{
Matsunori Nara ${ }^{1}$, Xiaoyi Zhao ${ }^{2}$ \\ ${ }^{1}$ Department of System Engineering, Tokyo University of Science, Suwa (TUS), 5000-1 Toyohira, Chino, Nagano, Japan \\ ${ }^{2}$ Course of Engineering and Management, Graduate school of Tokyo University of Science, Suwa, 5000-1 Toyohira, Chino, Nagano, Japan \\ Email: nara@rs.suwa.tus.ac.jp
}

Received 2012

\begin{abstract}
It is a technology that produces the hydrogen gases by using only the microorganism and sunlight from the wastewater to be at a loss because of processing. And, the produced hydrogen uses the fuel cell and is used to generate electricity. Because this technology doesn't use the organic matter that becomes food, and use the organic matter included in waste, clean power generation is possible. We researched the improvement of the hydrogen gas production efficiency. The purple non-sulfur photosynthesis bacillus was purely cultured, the substrate dependency was clarified, and the best substrate dosage was decided. Moreover, it was shown that the light wave length conversion net was effective for the hydrogen gas production efficiency improvement. On the other hand, a too strong light intensity showed becoming the growth obstruction of phototropic bacteria. When the light intensity for the photosynthesis is insufficient, the light wave length conversion net can support this. On the other hand, there was an effect of easing the trouble by light when the light intensity was excessive. We were able to offer the design parameter to produce the hydrogen gases with the wastewater efficiently.
\end{abstract}

Keywords: Wastewater Utilization; Hydrogen Gasproduction; Bio-mass Enrgy

\section{Introduction}

In this research, it is a purpose to develop the technology that manufactures hydrogen from sunlight with an organic wastewater and the photosynthetic microorganism. Because this technology doesn't use the organic matter that becomes food, and use the organic matter included in waste, clean power generation is possible. The maximum problem in the hydrogen gas production with the wastewater is that the energy production efficiency is low. We researched the improvement of the hydrogen gas production efficiency.

\section{Materials and Methods}

We developed the method of improving the ratio of an effective wavelength elements for the photosynthesis included in sunlight to improve photosynthetic microorganism's hydrogen production efficiency. That is, the material (wavelength conversion polymer resin) that was able to convert the ultraviolet rays of sunlight into the near-infrared radiation was made for trial purposes. This material is a product that adds the fluorescent dye (made by the Lumogem F RED-300 BASF company) to the polymer resin and molds it like the film. This is converted the absorbed light into the wavelength of a red belt (580-780 nm) that contributes to the photosynthesis, and fluoresces. The wavelength conversion material was molded like the net. The photosynthetic microorganism that had used it by the experiment was a mixed bacterial culture including Rhodopseudomonas Palustris. The ultraviolet rays lamp and sunlight were used as a light energy source supplied to the photosynthesis.
The photosynthetic microorganism that had purely cultured it experimented on the ultraviolet rays lamp as a source of light in the indoor temperature controlled bath.

And, the photosynthetic microorganism that had done the mixture culture did the experiment of which the source of light was sunlight in outdoor. Figure 1 showed the photograph of the pilot machine and the outline.

\section{Results and Discussion}

To culture photosynthetic microorganism (Rhodopseudomonas Palustris) purely first of all to design the hydrogen [san;u] system and to clarify an ecological characteristic, the [kainetei; kkusu] was analyzed. Figure 2 is showing of the specific growth rate of the photosynthetic microorganism in the pure culture system to the administered substrate quantity. Afterwards, the amount of an increase was saturated though the tendency that the number of microorganisms increases as the substrate quantity increased was observed. The relation between these shows the saturation curve, and it is understood that the enzyme reaction progresses. Then, to examine kinetic that hung
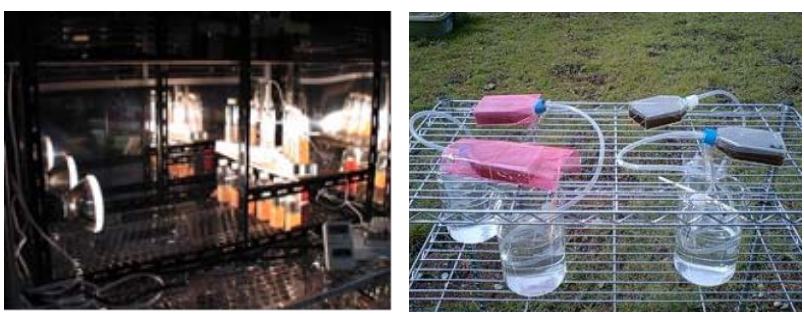

Figure 1. The photograph of the pilot machine and the outline. 


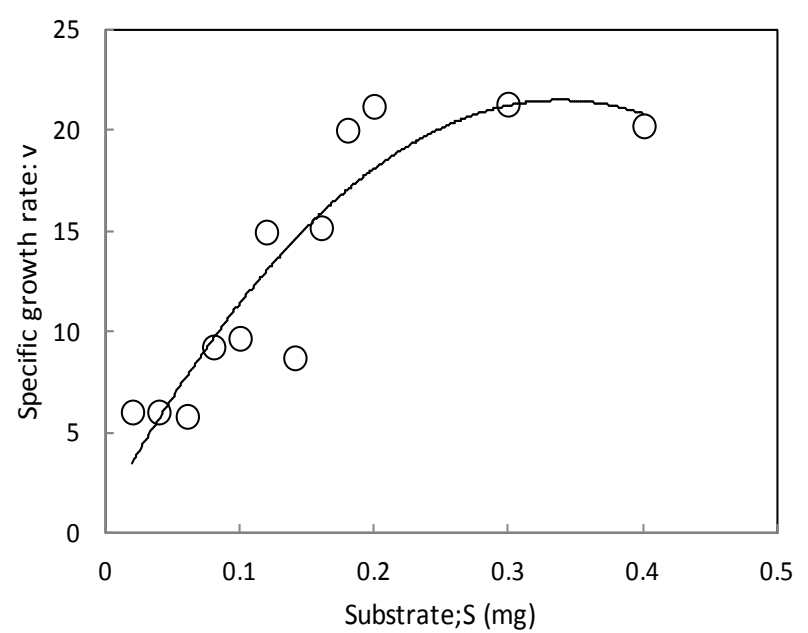

Figure 2. Relation between given subatrate and specific growthrate of microbe.

to the hydrogen production by the photosynthetic microorganism, Nanes-Woolf was plotted. Figure 3 showed the result of doing Hanes-Woolf Plot by thinking about the hydrogen production as an enzyme reaction. It seemed that the correlation coefficient of the regression line was about 0.66 , and an appropriate approximation. Vmax was about 31 as a result of the calculation and $\mathrm{Km}$ was 0.18 . Figure 4 are showing of the amounts of the hydrogen gas produced for the supplied substrate quantity (conversion of the amount of the carbohydrate). The volume of the reaction vessel was assumed to be $200 \mathrm{ml}$. The amounts of the hydrogen gas produced as the substrate quantity increases have increased. However, the gas production was indicated the maximum value, and decreased gradually around of the supply substrate amount ten grams afterwards. It has been understood that the optimum value exists in the substrate quantity supplied from this to the photosynthetic microorganism. Moreover, the amount of the gas generated in the source of artificial light was below the half of that of sunlight. Figure 5 showed the relation between the number of colonies of photosynthetic microorganisms that lived in the reaction vessel and the produced amount of the hydrogen gas. The amounts of the hydrogen gas produced as the microbial biomass increases have increased. However, the amounts of the gas produced with the hit for the number of fungus bodies to exceed 300 million have decreased. The upper bound of the microbial count in the actual experiment condition was presumed to be about 300 million pieces from the above-mentioned result. The substrate quantity administered in this case was 6.7 grams every the reaction vessel $200 \mathrm{ml}$. Moreover, when the source of artificial light was used, the hydrogen production was below the half in case of sunlight.

\section{Conclusion}

The experimental research into the improvement of the hydrogen production efficiency was done aiming to put the technology that produced the hydrogen gases with an organic waste fluid and the photosynthetic microorganism to practical use. First of all, the purple non-sulfur photosynthesis bacillus was purely cultured, the substrate dependency was clarified, and the

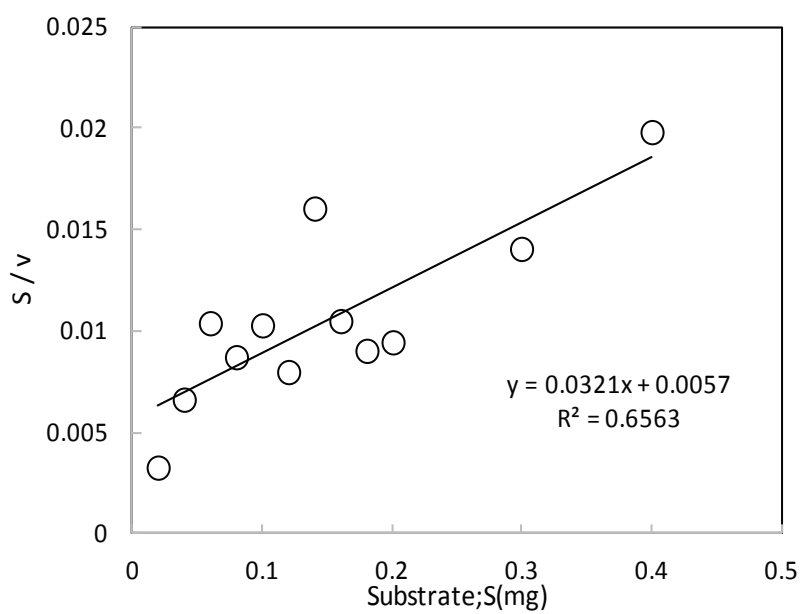

Figure 3. Hanes-Woolf plot in hydrogen production by photosynthetic microorganism.

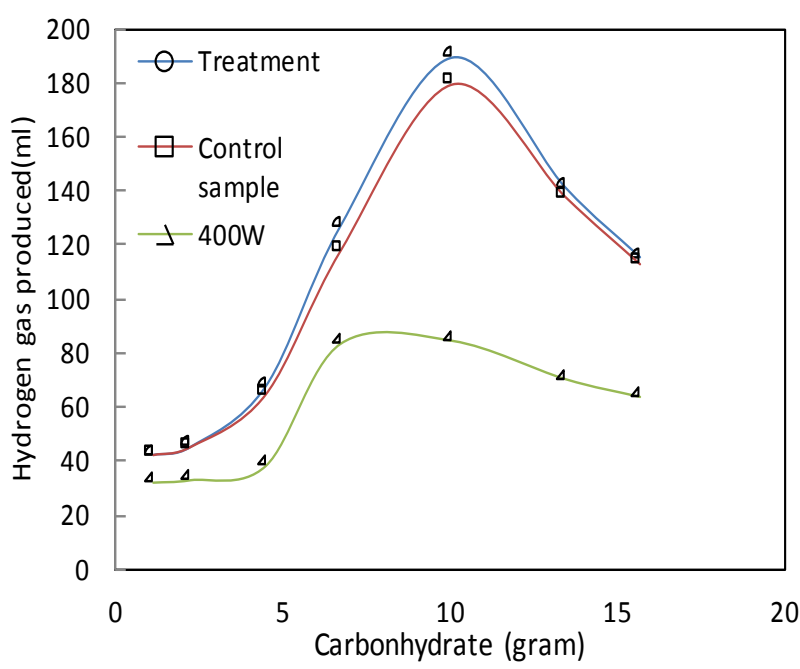

Figure 4. Relation between amount of given substrate and hydrogen gas production.

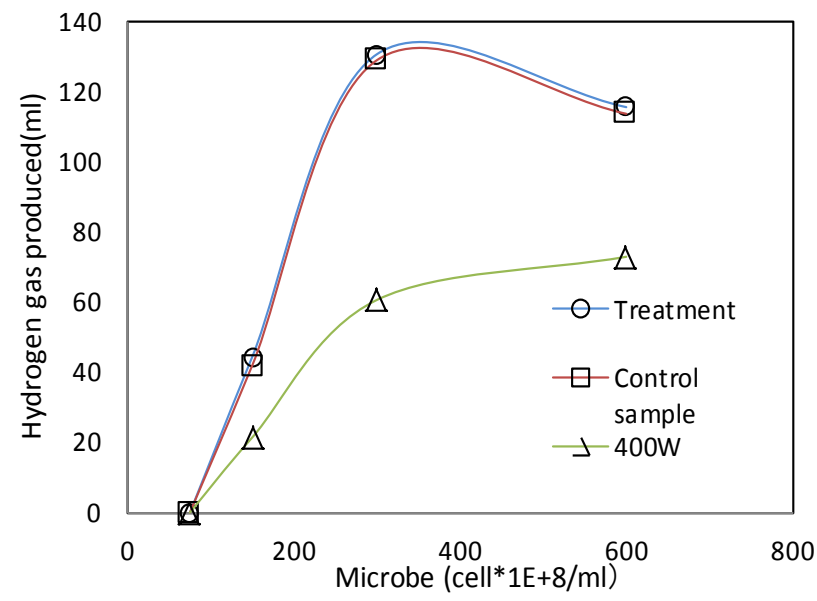

Figure 5. Relation between microbial count in the vessel and produced amount of hydrogen gas.

best substrate dosage was decided. Moreover, it was shown that 
the light wave length conversion net was effective for the hydrogen gas production efficiency improvement. On the other hand, a too strong light intensity showed becoming the growth obstruction of phototropic bacteria. When the light intensity for the photosynthesis is insufficient, the light wave length conversion net can support this. On the other hand, there was an effect of easing the trouble by light when the light intensity was excessive.

\section{REFERENCES}

[1] M.Nara: Research on improvement of hydrogen production efficiency by photosynthetic microorganism (Society of Heating, Air-Conditioning and Sanitary Engineers, Japan 2010)

[2] M.Nara, and H.Sugiura: Hydrogen gas production by anaerobic microorganism (Japan Society of Mechanical Engineers lecture collection 2009).

[3] H. Kitamura and others: Phototropic bacteria ( society publication center 2000) 